\title{
ANÁLISE DOS DADOS DE PREVALÊNCIA DE CÁRIE DENTAL NA CIDADE DE CAMPINAS, SP, BRASIL, DEPOIS DE DEZ ANOS DE FLUORAÇÃO DA ÁGUA DE ABASTECIMENTO PÚBLICO
}

\author{
Yvette VIEGAS *
}

Alfredo Reis VIEGAS *

RSPU-B/234

\begin{abstract}
VIEgas, Y. \& VIEgas, A. R. - Análise dos dados de prevalencia de cárie dental na cidade de Campinas, $S P$, Brasil, depois de dez anos de fluoração da água de abastecimento público. Rev. Saúde públ., S. Paulo, 8:399-409, 1974.

Resumo: Foram analisados os resultados dos dados de quatro levantamentos de prevalencia de cárie dental de crianças de 4 a 14 anos de idade da cidade de Campinas, SP, Brasil, com o objetivo de se verificar as reducóes de cárie dental encontradas nos dentes permanentes e primários durante o periodo de dez anos de fluoracão da água de abastecimento público. As reduçóes de prevalencia de carie observadas são similares às constatadas nos estudos pilotos dos Estados Unidos e do Brasil, sendo de $66 \%$ para os dentes permanentes $e$ de 53\% nos dentes primários. Nos grupos etários de 6 a 10 anos, $25 \%$ das criancas não têm nenhum dente primário atacado pela cárie, e $36 \%$ estão em igual condição em relação aos dentes permanentes.
\end{abstract}

Unitermos: Fluoração da água*; Cárie dental (prevenção) *; Cárie dental (prevalência)*; Campinas (S. Paulo - Brasil).

O presente trabalho tem por objetivo apresentar e analisar os dados de prevalência de cárie dental verificados no estudo de fluoração da áqua de abastecimento público na cidade de Campinas.

Campinas apresenta de um modo geral um clima ameno e saudável, com inverno seco. As temperaturas máximas têm como média $27^{\circ} \mathrm{C}$ e, as mínimas, $16^{\circ} \mathrm{C}$. Sua estação de tratamento de água é classificada como de $1 .^{2}$ classe e a água sofre tratamento completo. A população em 1962 era de 212.000 habitantes e em 1972 de 373.608.

Neste estudo de fluoração da água de abastecimento da cidade de Campinas, os primeiros dados foram coletados em junho de 1961 quando foi feito o levantamento de linha de base de prevalência de cárie dental.

A adição de flúor à água de abastecimento público foi iniciada em abril de 1962, e desde então tem sido adicionado ininterruptamente até a presente data (agosta-1974) .

Durante o período de 1962 a 1972 foram realizados três levantamentos, respectivamente nos anos de 1965, 1969 e 1972, a fim de avaliar os efeitos da fluoração da água de abastecimento público sobre a prevalência de cárie dental, comparan-

* Do Departamento de Prática de Saúde Pública da Faculdade de Saúde Pública da USP - Caixa Postal 8099 - São Paulo, SP - Brasil 
VIEGAS, Y. \& VIEGAS, A.R. - Análise dos dados de prevalência de cárie dental na cidade de Campinas, SP, Brasil, depois de dez anos de fluoração da água de abastecimento público. Rev. Saude puibl., S, Paulo, 8:399-409, 1974.

T A B E L A 1

Número total de crianças examinadas de 4 a 14 anos da idade, segundo o sexo, nos anos de 1961, 1965, 1969 e 1972 na cidade de Campinas, SP

\begin{tabular}{|c|c|c|c|c|c|c|c|c|}
\hline Ano & \multicolumn{2}{|c|}{1961} & \multicolumn{2}{|c|}{1965} & \multicolumn{2}{|c|}{1969} & \multicolumn{2}{|c|}{1972} \\
\hline Número & \multicolumn{2}{|c|}{12.576} & \multicolumn{2}{|c|}{5.051} & \multicolumn{2}{|c|}{4.874} & \multicolumn{2}{|c|}{9.606} \\
\hline $\mathrm{de}$ & MI & $\mathbf{F}$ & $\mathbf{M}$ & $\mathbf{F}$ & $\mathbf{M}$ & $F$ & $\mathrm{M}$ & $\mathbf{F}$ \\
\hline criancas & 6.081 & 6.495 & 2.580 & 2.471 & 2.502 & 2.372 & 4.791 & 4.815 \\
\hline
\end{tabular}

$M=$ masculino

$F=$ feminino

do os resultados observados com os encontrados no levantamento inicial.

0 número de crianças de 4 a 14 anos de idade examinadas nos quatro levantamentos pode ser constatado através da Tabela 1 .

O critério para a inclusão das crianças na respectiva idade foi a de anos completados.

As crianças examinadas dos 4 aos 14 anos de idade foram selecionadas através de amostragem sistemática entre as que estavam matriculadas nas escolas de ensino primário da rede escolar de Campinas.

Nos três últimos levantamentos só foram examinadas as crianças que viveram sempre em Campinas. Consideram-se como residentes contínuos todos os que durante suas vidas se ausentaram da cidade no máximo trinta dias por ano, seguindo-se o critério estabelecido por DEAN et al. ${ }^{4}$.

As crianças que foram examinadas nos quatro levantamentos, segundo a idade $\mathrm{e}$ o sexo, podem ser observadas através da Tabela 2.

Os exames foram feitos nas próprias escolas sob luz natural, usando-se explorador SSW n. ${ }^{\circ} 5$ e espelho plano.

Foram examinados os dentes irrompidos, classificando-os nas categorias correspondentes, segundo os índices utilizados e que foram o CPOD, e ceo, e o de primeiros molares permanentes.
Os critérios adotados nos quatro levantamentos foram sempre os mesmos e são os seguintes:

Dente irrompido - Os dentes foram considerados como irrompidos quando:

a) Os incisivos e os caninos apresentassem porções equivalente da coroa nos sentidos do longo eixo e do mesiodistal.

b) Os premolares apresentassem toda face oclusal exposta.

c) Os molares apresentassem pelo menos dois terços de sua superfície oclusal exposta.

Dente cariado - 0 dente foi considerado como cariado quando:

a) A lesão fosse clinicamente óbvia.

b) A opacidade do esmalte indicasse existência de cárie subjacente.

c) A extremidade da sonda exploradora pudesse penetrar até tecidos dentinário mole, que cedesse à pressão.

d) No caso de fissuras e cicatrículas, quando a sonda prendesse, suportasse seu próprio peso, e oferecesse resistência à sua retirada.

e) No caso do dente se encontrar com óxido de zinco e eugenol, ou com cimento de oxifosfato de zinco.

f) No caso de dente obturado mas com recidiva de cárie. 
VIEGAS Y. \& VIEGAS. A.P. - Análise dos dados de prevalênca de cárie dental na cidade de Campinas, SP. Brasil, depols de dez anos de fluoracão da água de abastecimento público. Rev. Sulde pliti S. Paulo, 8:399-409, 1974.

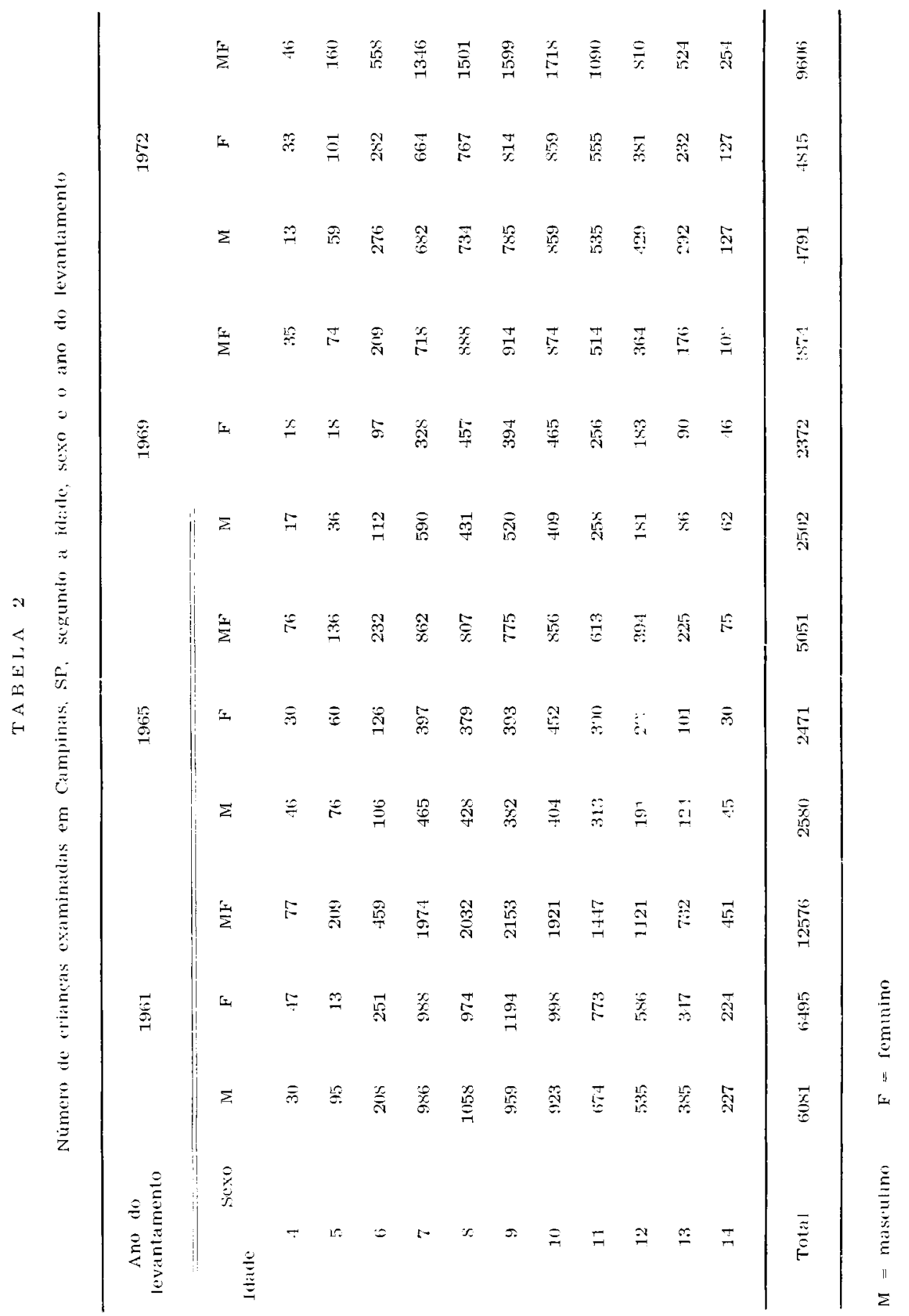


VIEGAS, Y. \& VIEGAS, A.R. - Análise cos dados de prevalência de cárie dental na cidade de Campinas, SP, Brasil, depois de dez anos de fluoração da água de abastecimento público. Rev. Saúde públ., S. Paulo, 8:399-409, 1974.

Dente obturado - $\mathrm{O}$ dente foi considerado como obturado quando:

a) Apresentasse restauração a ouro, acolite, amálgama, cimento de silicato, ou resina acrílica.

b) Apresentasse coroa total, ou pivot.

Dente extraido - $\mathrm{O}$ dente foi considerado como extraído quando:

a) Se encontrasse ausente da boca depois da época em que normalmente deveria ter irrompido.

Dente com extração indicada - $\mathbf{0}$ dente foi considerado com extração indicada quando:

a) Apresentasse uma lesão profunda, cuja polpa deveria ser exposta ao se fazer o preparo de cavidade.

b) Apresentasse uma lesão profunda com sinais de exposição pulpar.

Foram ainda levados em consideração os seguintes casos:

a) Os dentes ausentes devido a trauma por acidentes não foram computados como extraídos. b) Os dentes ausentes congenitamente bem como os extraídos para fins ortodônticos também não foram incluídos.

c) Foram excluídos do estudo as crianças com aparelhos ortodônticos fixos.

Todos os levantamentos foram precedidos de calibração dos examinadores, com - objetivo de que a interpretação dos critérios fosse correta, limitando-se desse modo o erro entre examinadores. Foram examinadores cirurgiôes dentistas do Serviço Dentário Escolar, treinados e supervisionados por um dos autores deste trabalho.

Os valores resultantes dos quatro levantamentos em relação ao índice $\mathrm{CPO} e$ seus componentes podem ser verificados nas Tabelas $3,4,5$ e 6 .

Como se pode observar, a prevalência de cárie dental foi diminuindo nas diferentes idades à medida que foi aumentando o número de anos de fluoração da água de abastecimento público.

Pode-se notar através da Tabela 7 que o percentual de redução de cárie dental no grupo etário de 5 a 6 anos de idade foi de ordem de $82 \%$ no grupo etário de 7 a 10 , de $58 \%$, no de 11 a 14 , de $50 \%$, e no de 7 a 14 , de $54 \%$.

T A B E L A 3

Indice CPO e componentes, segundo a idade, em 12.576 crianças de ambos os sexos da cidade de Campinas, SP, 1961

\begin{tabular}{rrrrrrrr}
\hline Idade & N.o de crianças & C & O & E & EI & CPO & TI \\
\hline 4 & 77 & 0.09 & - & - & - & 0.09 & 0.17 \\
5 & 209 & 0.37 & 0.02 & - & - & 0.39 & 1.26 \\
6 & 459 & 1.16 & 0.25 & 0.01 & 0.03 & 1.45 & 4.83 \\
7 & 1974 & 2.16 & 0.45 & 0.04 & 0.12 & 2.77 & 8.37 \\
8 & 2032 & 1.90 & 0.98 & 0.12 & 0.17 & 3.17 & 11.47 \\
9 & 2153 & 2.12 & 1.18 & 0.22 & 0.27 & 3.79 & 14.95 \\
10 & 1921 & 3.00 & 1.41 & 0.33 & 0.35 & 5.09 & 18.73 \\
11 & 1447 & 3.66 & 1.67 & 0.48 & 0.45 & 6.26 & 22.08 \\
12 & 1121 & 3.71 & 2.70 & 0.55 & 0.40 & 7.36 & 24.87 \\
13 & 732 & 4.01 & 4.14 & 0.54 & 0.47 & 9.16 & 26.37 \\
14 & 451 & 3.42 & 6.19 & 0.60 & 0.27 & 10.48 & 26.86 \\
\hline C & & & & & & &
\end{tabular}


VIEGAS, Y. \& VIEGAS, A.R. - Análise dos dados de prevalência de cárie dental na cidade de Campinas, SP, Brasil, depois de dez anos de fluoração da água de abastecimento público, Rev. Saúde públ., S. Paulo, 8:399-409, 1974.

TA B E I A 4

Indice CPO e componentes, segundo a idade, em 5051 crianças de ambos os sexos, da cidade de Campinas, SP, 1965

\begin{tabular}{|c|c|c|c|c|c|c|c|}
\hline Idade & N. de crianças & $\mathrm{C}$ & $\mathrm{O}$ & $\mathbf{E}$ & EI & $\mathrm{CPO}$ & TI \\
\hline 4 & 76 & 一 & - & - & 一 & - & - \\
\hline 5 & 136 & 0.02 & - & - & 一 & 0.02 & 0.60 \\
\hline 6 & 232 & 0.73 & 0.21 & 一 & 0.01 & 0.95 & 5.45 \\
\hline 7 & 862 & 1.04 & 0.74 & 0.02 & 0.02 & 1.82 & 8.25 \\
\hline 8 & 807 & 1.50 & 1.09 & 0.07 & 0.09 & 2.75 & 11.40 \\
\hline 9 & 775 & 1.69 & 1.42 & 0.19 & 0.17 & 3.47 & 14.48 \\
\hline 10 & 856 & 2.10 & 1.70 & 0.36 & 0.25 & 4.41 & 18.27 \\
\hline 11 & 613 & 2.83 & 1.89 & 0.48 & 0.34 & $\mathbf{5 . 5 4}$ & 21.82 \\
\hline 12 & 394 & 3.63 & 2.23 & 0.67 & 0.42 & 6.95 & 24.27 \\
\hline 13 & 225 & 4.47 & 3.08 & 0.71 & 0.62 & 8.88 & 25.84 \\
\hline 14 & 75 & 3.78 & 3.85 & 0.71 & 0.47 & 8.81 & 23.20 \\
\hline
\end{tabular}

TABELA 5

Indice CPO e componentes, segundo a idade, em 4874 crianças de ambos os sexos, da cidade de Campinas, SP, 1969

\begin{tabular}{|c|c|c|c|c|c|c|c|}
\hline Idade & N. ${ }^{\circ}$ e crianças & $\mathrm{C}$ & O & $\mathrm{E}$ & $\mathrm{EI}$ & $\mathrm{CPO}$ & TI \\
\hline 4 & 35 & - & 一 & - & 一 & 一 & - \\
\hline 5 & 74 & 0.14 & - & - & 一 & 0.14 & 1.35 \\
\hline 6 & 209 & 0.23 & 0.12 & 0.004 & 一 & 0.36 & 5.09 \\
\hline 7 & 718 & 0.44 & 0.68 & 0.02 & 0.03 & 1.17 & 8.94 \\
\hline 8 & 888 & 0.57 & 1.04 & 0.02 & 0.04 & 1.67 & 12.01 \\
\hline 9 & 914 & 0.75 & 1.56 & 0.07 & 0.05 & 2.43 & 14.24 \\
\hline 10 & 874 & 0.89 & 2.07 & 0.09 & 0.07 & 3.12 & 18.79 \\
\hline 11 & 514 & 1.25 & 2.61 & 0.14 & 0.09 & 4.09 & 22.67 \\
\hline 12 & 364 & 1.38 & 3.38 & 0.24 & 0.18 & 5.13 & 25.41 \\
\hline 13 & 176 & 1.79 & 4.06 & 0.30 & 0.16 & 6.31 & 26.66 \\
\hline 14 & 108 & 1.60 & 4.69 & 0.27 & 0.30 & 6.86 & 27.10 \\
\hline
\end{tabular}

T A B E L A 6

Indice CPO e componentes, segundo a idade, em 9606 crianças de ambos os sexos, da cidade de Campinas, SP, 1972

\begin{tabular}{rrrrrrrr}
\hline Idade & N.O de crianças & $\mathrm{C}$ & $\mathrm{O}$ & $\mathrm{E}$ & $\mathrm{EI}$ & $\mathrm{CPO}$ & TI \\
\hline 4 & 46 & - & $-\overline{01}$ & - & - & - & 0.04 \\
5 & 160 & 0.01 & 0.01 & - & 0.04 & 1.44 \\
6 & 558 & 0.24 & 0.14 & - & - & 0.38 & 5.65 \\
7 & 1346 & 0.35 & 0.44 & 0.01 & 0.01 & 0.81 & 9.02 \\
8 & 1501 & 0.40 & 0.97 & 0.01 & 0.02 & 1.40 & 11.73 \\
9 & 1599 & 0.47 & 1.31 & 0.04 & 0.04 & 1.86 & 14.15 \\
10 & 1718 & 0.49 & 1.69 & 0.04 & 0.06 & 2.28 & $\mathbf{1 8 . 1 0}$ \\
11 & 1090 & 0.51 & 2.18 & 0.07 & 0.07 & 2.83 & 22.52 \\
12 & 810 & 0.59 & 2.79 & 0.13 & 0.12 & 3.63 & 24.99 \\
13 & 524 & 0.71 & 3.20 & 0.21 & 0.12 & 4.24 & 25.53 \\
14 & 254 & 0.54 & 4.55 & 0.22 & 0.10 & 5.41 & 27.30 \\
\hline
\end{tabular}


VIEGAS, Y. \& VIEGAS, A.R. - Análise dos dados de prevalência de cárie dental na cidade de Campinas, SP, Brasil, depois de dez anos de fluoração da água de abastecimento público. Rev. Saúde públ., S. Paulo, 8:399-409, 1974.

T A B ELA T

CPO médio e percentagens de redução em ambos os sexos, segundo a idade observados no periodo de 1961 a 1972 na cidade de Campinas, SP

\begin{tabular}{|c|c|c|c|c|c|c|c|}
\hline Ano & 1961 & & 1965 & & 1969 & & 1972 \\
\hline Idade & $\overline{\mathrm{CPO}}$ & $\overline{\mathrm{CPO}}$ & $\begin{array}{l}\text { Percentual } \\
\text { de redução }\end{array}$ & $\overline{\mathrm{CPO}}$ & $\begin{array}{l}\text { Percentual } \\
\text { de redução }\end{array}$ & $\overline{\mathrm{CPO}}$ & $\begin{array}{l}\text { Percentual } \\
\text { de redução }\end{array}$ \\
\hline 5 & 0.39 & 0.02 & 94.87 & 0.14 & 64.10 & 0.04 & 89.74 \\
\hline 6 & 1.45 & 0.95 & 34.48 & 0.36 & 75.17 & 0.38 & 73.79 \\
\hline 7 & 2.77 & 1.82 & 34.29 & 1.17 & 57.71 & 0.81 & 70.76 \\
\hline 8 & 3.17 & 2.75 & 13.25 & 1.67 & 47.31 & 1.40 & 55.84 \\
\hline 9 & 3.79 & 3.47 & 8.44 & 2.43 & 35.88 & 1.86 & 50.92 \\
\hline 10 & 5.09 & 4.41 & 9.13 & 3.12 & 38.70 & 2.28 & 55.21 \\
\hline 11 & 6.26 & 5.54 & 9.11 & 4,09 & 34.66 & 2.83 & 45.20 \\
\hline 12 & 7.36 & 6.95 & 9.05 & 5.18 & 29.62 & 3.63 & 50.68 \\
\hline 13 & 9.16 & 8.88 & 3.05 & 6.31 & 31.11 & 4.24 & 53.71 \\
\hline 14 & 10.48 & 8.81 & 15.94 & 6.86 & 34.54 & 5.41 & 48.37 \\
\hline
\end{tabular}

Se compararmos os dados de 10 anos de Campinas (Tabela 9) com os estudos pilotos dos Estados Unidos 2 (Newburgh-Kingston e Grand Rapids-Muskegon) e do Brasil * (Baixo Guandu), pode-se verificar que as reduçôes encontradas são similares às obtidas nesses estudos, equi- valendo-se às observadas em Newhurgh e Grand Rapids, sendo ligeiramente menores que as verificadas na cidade de Baixo Guandu, mas não apresentando em nenhum caso diferenças estatisticamente significantes.

T A B E L A 8

Comparação de percentual de redução da prevalência de cárie dental em dentes permanentes das crianças da cidade de Campinas, SP, com as de Newburgh e Grand Rapids. USA e I3aixo Guandu, ES, após 10 anos de fluoração

\begin{tabular}{|c|c|c|c|c|c|}
\hline \multirow{2}{*}{$\begin{array}{l}\text { Grupo } \\
\text { etário }\end{array}$} & \multicolumn{5}{|c|}{$C i d a d e s$} \\
\hline & Campinas & Newburgh & Grand Rapids & Baixo & Guandu \\
\hline $61-19$ & 63 & 58 & 61 & & -- \\
\hline $7: 10$ & 58 & - & 56 & & 64 \\
\hline $10 !-12$ & 50 & 53 & 53 & & 58 \\
\hline $13:-14$ & 51 & 48 & 48 & & 53 \\
\hline $7|-| 14$ & 54 & - & 52 & & 59 \\
\hline $11-14$ & 50 & - & 48 & & 55 \\
\hline
\end{tabular}


VIEGAS, Y. \& VIEGAS, A.R. - Análise dos dados de prevalêncía de cárie dental na cidade de Campinas, SP, Brasil, depois de dez anos de fluoração da água de abastecimento público. Rev. Saúde públ., S. Paulo, 8:399-409, 1974.

T A B E L A 9

ceo médio e percentagens de reducão em ambos os sexos, segundo a idade, observados no periodo de 1961 a 1972 na cidade de Campinas, SP

\begin{tabular}{|c|c|c|c|c|c|c|c|}
\hline Ano & 1961 & \multicolumn{2}{|c|}{1965} & \multicolumn{2}{|c|}{1969} & \multicolumn{2}{|c|}{1972} \\
\hline Idade & ceo & ceo & $\begin{array}{l}\text { Percentual } \\
\text { de redução }\end{array}$ & ceo & $\begin{array}{l}\text { Percentual } \\
\text { de redução }\end{array}$ & ceo & $\begin{array}{l}\text { Percentual } \\
\text { de reducão }\end{array}$ \\
\hline 4 & 5.06 & 3.57 & 29 & 1.65 & 67 & 0.86 & 83 \\
\hline 5 & 5.48 & 4.56 & 17 & 2.40 & 56 & 1.75 & 68 \\
\hline 6 & 6.17 & 5.69 & 8 & 2.55 & 59 & 2.55 & 59 \\
\hline 7 & 5.91 & 5.74 & 3 & 2.81 & 52 & 2.65 & 55 \\
\hline 8 & 4.18 & 4.62 & - & 2.91 & 30 & 2.45 & 41 \\
\hline 9 & 2.85 & 3.06 & - & 2.34 & 18 & 1.88 & 34 \\
\hline 10 & 1.64 & 1.65 & - & 1.36 & 17 & 1.13 & 31 \\
\hline 11 & 0.77 & 0.74 & 4 & 0.58 & 25 & 0.58 & 25 \\
\hline 12 & 0.26 & 0.29 & - & 0.18 & 31 & 0.20 & 23 \\
\hline 13 & 0.07 & 0.12 & - & 0.05 & 29 & 0.07 & 0 \\
\hline 14 & 0.03 & 0.09 & - & 0.04 & - & 0.00 & 100 \\
\hline
\end{tabular}

As maiores reduções observadas no estudo do Baixo Guandu, cremos, podem ter sua possível explicação em um destes fatos:

1. Há um controle muito rígido na seleção das crianças que são examinadas no Baixo Guandu. Isto foi possivel nesse estudo porque a população da cidade é pequena, e o Serviço Especial de Saúde Pública (F.SESP) possuindo unidade sanitária nessa cidade, que inclui um programa de saúde da boca, tem cadastrada praticamente toda a população, e pode verificar realmente quais as crianças que viveram sempre na cidade usufruindo assim o benefício completo da ação do flúor através da injestão da água de abastecimento público. Nos outros estudos, esse controle por melhor que tenha sido feito, devem ter ocorrido falhas, daí as reduções observadas possivelmente terem sido ligeiramente menores, contudo não fossem as diferenças estatisticamente significantes.

2. Os examinadores que participaram do estudo do Baixo Guandu possuem um nível de calibração muito superior ao de Campinas.

A prevalência de cárie dental nos dentes da primeira dentição também foi reduzida dentro do esperado, como pode ser notado através dos dados da Tabela 9 .

Verifica-se que a redução no grupo etário de 5 a 10 anos de idade que recebeu o benefício durante todo o período de vida foi da ordem de $66 \%$. Esse fato é importante pois a prevalência de deformidades dento faciais se reduz à medida que é conservado o comprimento do arco dental.

Hill et al. observaram crianças de 6 a 8 anos de idade que foram divididas em dois grupos. Num deles houve perda 
VIEGAS, Y. \& VIEGAS, A.R. - Análise dos dados de prevalência de cárie dental na cidade de Campinas, SP, Brasil, depois de dez anos de fluoração da água de abastecimento público. Rev. Saúde públ., S. Paulo, 8:399-409, 1974.

precoce dos segundos molares primários, e no outro não. A prevalência de más oclusões foi de ordem de $45,77 \%$ para o primeiro e de $31 \%$ para o segundo, dando uma diferença de $14,77 \%$ entre ambos.

MCCALL ${ }^{6}$ observando crianças de 2 a 6 anos rotou a presença de $52 \%$ de casos de má oclusão nas crianças que sofreram extrações precoces de molares primários, e naquelas que não foram submetidas a extrações notou apenas $32 \%$ de casos com má oclusão, sendo a diferença entre ambos da ordem de $20 \%$.

As conseqüências decorrentes da perda de comprimento de arco acarretam segundo esses estudos um aumento de más oclusões que varia de 15 a $20 \%$.

Com a fluoração, há redução da prevalência da cárie dental, e pelas observações do estudo de Evanston realizadas por HiLl et al. ${ }^{5}$ notou-se uma redução de casos de má oclusão no grupo etário de 6 a 8 anos da ordem de $8 \%$, e no de 12 a 14, de 9,5\%. No estudo de Newburgh-Kingston Ast et al. ${ }^{1}$, ao compararem crianças de 13 a 14 anos de idade, verificaram uma diferença de $13,1 \%$ a menos de casos de má oclusão de carater incapacitante nas crianças de Newburgh (com fluoração) quando comparadas às de Kingston (sem fluoração).

Outro dado que deve ser assinalado é o relativo ao ataque de cárie dos primeiros molares permanentes. $O$ ataque desse dente representa um "backlog" nas idades que são atendidas prioritariamente nos programas de saúde da boca. A redução de seu ataque, diminuindo desse modo as necessidades acumuladas, permite um aumento significativo na cobertura da população a ser tratada.

Na Tabela 10 pode-se observar o CPO médio dos primeiros molares permanentes antes e depois de dez anos de fluoração. Verifica-se que a redução no grupo etário de 6 a 10 anos foi de $50,75 \%$.
TABELA 10

CPO médio dos primeiros molares permanentes e percentagem de redução em crianças de ambos os sexos, segundo a idade, observados no periodo de 1961-1972 - Campinas, SP

\begin{tabular}{|c|c|c|c|}
\hline Ano & 1961 & 1972 & \multirow{3}{*}{$\begin{array}{c}\text { Percentual } \\
\text { de } \\
\text { redução }\end{array}$} \\
\hline \multirow{2}{*}{ Idade } & $\overline{\mathrm{CPO}}$ & $\overline{\mathrm{CPO}}$ & \\
\hline & $10^{\circ} \mathrm{s.M}$ & $1.0 \mathrm{s.M}$ & \\
\hline 5 & 0.78 & 0.13 & 83.34 \\
\hline 6 & 1.58 & 0.52 & 67.09 \\
\hline 7 & 2.66 & 0.83 & 68.80 \\
\hline 8 & 2.84 & 1.38 & 51.41 \\
\hline 9 & 2.93 & 1.77 & 39.59 \\
\hline 10 & 3.31 & 2.05 & 38.07 \\
\hline 11 & 3.42 & 2.32 & 32.16 \\
\hline 12 & 3.54 & 2.59 & 26.84 \\
\hline 13 & 3.79 & 2.59 & 31.66 \\
\hline 14 & 3.84 & 2.92 & 23.96 \\
\hline
\end{tabular}

Paralelamente observa-se também um aumento da percentagem de crianças com primeiros molares permanentes sem cárie. Através dos dados da Tabela 11 pode-se verificar as mudanças que ocorreram depois de 10 anos de fluoração no estudo de Campinas.

T A B E L A 11

Percentagem de crianças de ambos os sexos, segundo a idade, com os primeiros molares permanentes sem cárie observada no periodo 1961-1972 - Campinas, SP

\begin{tabular}{|c|c|c|c|}
\hline Ano & 1961 & 1972 & \multirow{2}{*}{$\begin{array}{c}\text { Diferença } \\
\text { de } \\
\text { percentuais }\end{array}$} \\
\hline Idade & \multicolumn{2}{|c|}{$\begin{array}{l}\text { Percentual de } \\
\text { crianças com } \\
1 .^{\circ} \mathrm{s} . \mathrm{M} \text { sem cárie }\end{array}$} & \\
\hline 5 & 34.78 & 94.23 & 59.45 \\
\hline 6 & 30.61 & 65.08 & 34.47 \\
\hline 7 & 14.51 & 63.55 & 49.04 \\
\hline 8 & 12.10 & 46.89 & 34.79 \\
\hline 9 & 9.59 & 35.40 & 25.81 \\
\hline 10 & 3.86 & 29.63 & 25.77 \\
\hline 11 & 4.30 & 23.39 & 19.09 \\
\hline 12 & 3.09 & 18.15 & 15.06 \\
\hline 13 & 0.41 & 14.28 & 13.87 \\
\hline 14 & 0.00 & 11.81 & 11.81 \\
\hline
\end{tabular}


VIEGAS, Y. \& VIEGAS, A.R. - Análise dos dados de prevalência de cárie dental na cidade de Campinas, SP, Brasil, depois de dez anos de fluoraçáo da água de abastecimento público. Rev. Saúde públ., S. Paulo, 8:399-409, 1974.

$O$ aumento de crianças com primeiros molares permanentes sem cárie no grupo etário de 6 a 10 anos foi de $33,98 \%$; todavia o aumento observado nas crianças de 11 a 12 anos foi menor. Esse fato ocorre porque as crianças dessas idades no início do estudo estavam incluídas na faixa etária de um dia a 2 anos incompletos, e assim sendo seus primeiros molares permanentes não foram beneficiados "in totum" pelo flúor, sendo que este benefício foi tanto menor quanto maior a idade da criança ao ser iniciado o estudo, dependendo do estágio de calcificação do esmalte em que se encontravam. Por esta razão o ataque de cárie dos primeiros molares permanentes foi ainda alto, e por isso não acompanhou de modo similar a redução do ataque total de cárie de todos os dentes permanentes irrompidos (CPO observado), pois destes, excepto os primeiros molares, os outros já foram beneficiados pelo flúor durante a calcificação, sendo essa a razão de se observar um maior percentual de redução do índice CPO nas crianças de 11 a 12 anos do que o verificado para os primeiros molares permanentes.

É lógico que se se fizer um novo levantamento em 1974, isso não mais ocorrerá, pois aí as crianças de 11 a 12 anos de idade já terão recebido o benefício completo do flúor, e então o ataque dos primeiros molares permanentes será menor, e estará proporcional ao ataque de todos os dentes.

Convém lembrar que a ação do flúor, segundo BRUDEVOLD ${ }^{3}$, ocorre em três etapas: durante a calcificação, atuando no corpo do cristal da hidróxidoapatita; antes da erupção, atuando na superfície do esmalte; e depois da erupção, atuando na superfície do esmalte. Logo o benefício expresso através da redução observada nos primeiros molares das crianças de 11 a 14 anos se deve à ação do flúor nas duas últimas etapas.
Outro evento que foi observado no estudo de Campinas e que merece ser assinalado é o aumento que ocorreu no número de crianças sem nenhum dente atacado pela cárie, isto é, isentas de cárie.

$\mathrm{Na}$ Tabela 12 pode-se observar a percentagem de crianças com ceo zero, e na Tabela 13 , a percentagem de crianças com CPO zero.

TABELA 12

Percentagem de crianca, de ambos os sexos e segundo a idade, sem cárie nos dentes da primeira dentição observada no periodo de 1961-1972 - Campinas, SP

\begin{tabular}{|c|c|c|c|}
\hline Ano & 1961 & 1972 & \multirow{2}{*}{$\begin{array}{c}\text { Diferença } \\
\text { de } \\
\text { percentuals }\end{array}$} \\
\hline Idade & \multicolumn{2}{|c|}{$\begin{array}{l}\text { Percentual de } \\
\text { crianças com } \\
\text { ceo zero }\end{array}$} & \\
\hline 4 & 21.67 & 76.09 & 54.42 \\
\hline 5 & 12.00 & 50.62 & 38.62 \\
\hline 6 & 5.35 & 32.43 & 27.08 \\
\hline 7 & 2.58 & 29.29 & 26.71 \\
\hline 8 & 5.52 & 26.93 & 21.41 \\
\hline 9 & 8.54 & 33.02 & 24.48 \\
\hline 10 & 12.27 & 37.96 & 25.69 \\
\hline 11 & 13.07 & 37.30 & 24.23 \\
\hline 12 & 20.43 & 36.49 & 16.06 \\
\hline 13 & 15.38 & 25.71 & 10.33 \\
\hline 14 & 0.00 & 33.33 & 33.33 \\
\hline $61-10$ & 6.85 & 31.93 & 25.07 \\
\hline $6|-| 14$ & 9.24 & 32.49 & 23.26 \\
\hline
\end{tabular}

Como se nota, $25 \%$ das crianças não têm nenhum dente da primeira dentição atacado pela cárie no grupo etário de 6 a 10 anos, e 23\%, no de 6 a 14 anos.

Verifica-se em relação aos dentes permanentes que a percentagem de crianças sem nenhum dente atacado pela cárie, no grupo etário de 6 a 10 anos, é de $36 \%$, e $26 \%$ no grupo de 6 a 14 anos (Tabela 13).

No estudo do Baixo Guandu observou-se que $27 \%$ das crianças de 7 a 14 anos estavam isentas de cárie em relação aos dentes permanentes, enquanto em Campi- 
VIEGAS, Y. \& VIEGAS, A.R. - Análise dos dados de prevalência de cárie dental na cidade de Campinas, SP. Brasil, depois de dez anos de fluoracão da água de abastecimento público. Rev. Salude pübl,. S. Paulo, 8:399-409, 1974.

T A B E L A 13

Percentagem de criança, de ambos os sexos e sezundo a idade, sem cárie nos dentes permanentes observada no periodo de 19611972 - Campinas, SP

\begin{tabular}{|c|c|c|c|}
\hline Ano & 1961 & 1972 & \multirow{2}{*}{$\begin{array}{c}\text { Diferenca } \\
\text { de } \\
\text { percentuais }\end{array}$} \\
\hline Idade & \multicolumn{2}{|c|}{$\begin{array}{l}\text { Percentual de } \\
\text { criancas com } \\
\text { CPO zero }\end{array}$} & \\
\hline 5 & 50.98 & 95.15 & 45.17 \\
\hline 6 & 36.51 & 78.79 & 42.28 \\
\hline 7 & 14.39 & 63.11 & 48.72 \\
\hline 8 & 9.52 & 45.56 & 36.04 \\
\hline 9 & 6.15 & 35.36 & 29.21 \\
\hline 10 & 3.78 & 29.50 & 25.72 \\
\hline 11 & 3.13 & 23.65 & 20.52 \\
\hline 12 & 3.08 & 17.04 & 13.96 \\
\hline 13 & 1.78 & 13.69 & 11.91 \\
\hline 14 & 0.07 & 9.84 & 9.77 \\
\hline $6 \mid-10$ & $14.0 \%$ & 50.46 & 36.39 \\
\hline $6:-14$ & 8.71 & 35.17 & 26.46 \\
\hline
\end{tabular}

nas, nesse grupo etário, observou-se uma percentagem de $24 \%$

Esse dado é muito significativo, pois mostra que aproximadamente um quarto das crianças em idade escolar não apresenta nenhuma necessidade de tratamento relacionado ao problema da cárie dental na dentição permanente.

Outro aspecto que merece ser ressaltado é a melhoria da relação entre dentes extraídos e obturados. Em 1961 essa relação era de 1 dente extraído para 5,74 obturados, tendo passado em 1972 para a de 1 extraído para cada 23 obturados, o que representa uma melhoria de $75 \%$.

Fato análogo se observa também ao verificarmos a relação entre dentes cariados mais obturados com os dentes extraídos mais os com extração indicada. Essa relação em 1961 era de 1 E + EI para 7,46 $\mathrm{C}+0$, e em 1972 passou a $1 \mathrm{E}+\mathrm{EI}$ para 24,62 C +0 , o que indica uma melhoria de $70 \%$.

$$
\text { CONCLUSOES }
$$

Os principais fatos detectados no estudo de fluoração de Campinas são:

1. As reduções observadas na prevalência $d \epsilon^{-}$cárie dental são similares às obtidas aos estudos pilotos da América do Norte e as do Brasil.

2. O grupo etário de 5 a 10 anos de idade que recebeu benefício de flúor durante todo o período de vida apresentou uma redução da ordem de $66 \%$ em relação aos dentes permanentes.

3. A redução de cárie observada em relação aos dentes primários no grupo etário de 4 a 10 anos de idade foi de $53 \%$.

4. O ataque de cárie dos primeiros molares permanentes mostra no grupo de 6 a 10 anos de idade uma redução de $50,75 \%$.

5. Na dentição primária $25 \%$ das crianças de 6 a 10 anos de idade não apresentam nenhum dente atacado pela cárie.

6. Na dentição permanentes $36 \%$ das crianças de 6 a 10 anos de idade não têm dente atacado pela cárie.

7. A proporção de dentes extraídos para obturados apresenta uma melhoria de $75 \%$, e a de extraídos + extração indicada para cariados + obturados, de $70 \%$. 
VIEGAS, Y. \& VIEGAS, A.R. - Análise cios la los tie puralenc a de carie dental na curade de Campinas, SP, Brasil, depois de dez anos de fluoiaḉo da água de abastecimento pú๖ico. Rev. Saúde pübl.. S. Paulo, 8:399-403, $197:$

VIEGAS, Y \& VIEGAS, A. R. - LData analysis of the wrevalence of dental caries in Campinas city (S. Paulo, Brazll) after ten years of water fluoridation.] Rev. Saúde púbi., S. Paulo, 8:309-409, 1934.

SUMMARY: Data of four surveys of dental curies prevalence of children If to 14 years old in Campinas city (S. Paulo State, Brazil) were analysed to verify the reductions observed in the permant ni and primary teeth during the period of ten years of water fluordation. The reductions of dental caries prevalence observed are similar to the ones obtained in the pilot studies done in USA and Brazil, and were of $66 \%$ in the permanent dentition and $53 \%$ in the primary one. In the age grouns of 6 to $10,25 \%$ of children do not have any primary teeth attacked by caries, and $36 \%$ are in equal condition as regards to permanent teeth.

UNITERMS: Water fluoridation*; Dental curies prevention*; Dental caries prevalence*

\section{REFERENCIAS BIBLIOGRAFICAS}

1. AST, D.B. et al. - Prevalence of maloc. clusion, related to dental caries and lost first permaneni molars, in a fluoridated city and a fluoride-deficient city. Amer. J. Orthodont., 48: $106-12, \quad 1962$

2. AST, D.B. \& FITZGERALD, B. - Effactiveness of water fluoridation. $J$. Amer. dent. Ass, 65:581-7, 1962.

3. BRUDEVOLD, F. - II Chemical composition of the teeth in relation to caries. In: SOGNNAES, R.F. Chemistry and prevention of dental caries. Springfield, Charles $\mathrm{C}$. Thomas, 1962. p. 32-88.

4. DEAN, H.T. ?: al. - Mottled enamel in Texas. Publ. Hlth Rep., 50:424-12, 1935.
5. HILL, I.N. et al. - The Evanston dental caries study. XIX. Prevalence of malocelusion of children in a fluoridated and control area. J. dent. Res. $38: 782-94,1959$.

6. MAC CALL, J.O. - A study of malocclusion in preschool and school children. Dent. Itens Interest., 65:43-8, 1943.

7. VIEGAS, A.R. - Indice simplificado para estimar a prevalência de cárie cental em criancas de 7 a 12 anos cle idade, São Paulo, 1968. [Tese de cátedra - Faculdade de saúde Pública, USP].

Recebido para publicação em 16/9/197/ Aprotado para publicaço em 9/10/197/ 